\title{
The Optimization of a Virtual Dual Production-Inventory System under Dynamic Supply Disruption Risk
}

\author{
Yu Chen, ${ }^{1}$ Liyuan Liu $\mathbb{D D}^{2}{ }^{2}$ Victor Shi, ${ }^{3}$ Yibin Zhang, ${ }^{1,4}$ and Jing $\mathrm{Zhu}^{5}$ \\ ${ }^{1}$ School of Business Administration, Shanghai Lixin University of Accounting and Finance, Shanghai 201620, China \\ ${ }^{2}$ School of Mathematics Physics and Statistics, Shanghai University of Engineering Science, Shanghai 201620, China \\ ${ }^{3}$ Lazaridis School of Business and Economics, Wilfrid Laurier University, Waterloo, ON N2L 3C5, Canada \\ ${ }^{4}$ Institute for Manufacturing Manufacturing and Management Division, Department of Engineering, University of Cambridge, \\ 17 Charles Babbage Rd, Cambridge, CB3 OFS, UK \\ ${ }^{5}$ School of Business Administration, Southwestern University of Finance and Economics, Chengdu 611130, Sichuan, China
}

Correspondence should be addressed to Liyuan Liu; 21180014@sues.edu.cn

Received 5 May 2020; Revised 10 November 2020; Accepted 13 December 2020; Published 24 December 2020

Academic Editor: Jos Manuel Gal n

Copyright $\odot 2020$ Yu Chen et al. This is an open access article distributed under the Creative Commons Attribution License, which permits unrestricted use, distribution, and reproduction in any medium, provided the original work is properly cited.

Major events such as the COVID-19 pandemic, Olympic Games, and G20 Summit bring about supplier disruption risks and challenges to supply chain management. To help deal with these risks, a virtual dual-sourcing production-inventory system can be deployed. In this paper, we study such a system which consists of a raw material supplier, a manufacturer, and a virtual dualsourcing contingency supplier. The manufacturer needs to determine the production, procurement, and inventory plan of raw materials. When its supplier is interrupted, the manufacturer may need to adjust the production and inventory plan and work with the contingency supplier. We develop a system dynamics method to simulate the operations in this production-inventory system to identify the approximately optimal order-up-to-level inventory policies. We find that the virtual dual production-inventory strategy can be the optimal contingency policy to deal with supplier dynamic disruption risks. Furthermore, for disruption risk with low frequency and long duration, the manufacturer should increase the safety inventory level before the disruption. Otherwise, it should increase the safety inventory level in every cycle.

\section{Introduction}

As the COVID-19 pandemic of 2020 continues to unfold, lockdowns of countries and cities have caused severe disruptions in various supply chains such as healthcare and food supply chains [1]. This led to supply disruption for essential products such as food, medical testing kits, and PPEs [2]. In these supply chains, a recovery and resiliency plan should be considered for supply-side disruptions. Moreover, large-scale urban events such as G20 and Summer Olympics games can cause supply disruption risks to firms' normal operations including raw material procurement, production, and distribution [3]. To deal with these disruption risks, firms often build extra inventory or maintain backup suppliers.

Also, unpredictable supply disruptions such as natural or manmade disasters and accidents can severely impact supply chains. For example, on March 17, 2000, a lightning caused a factory fire at a plant of Royal Philips Electronics Company located in New Mexico, USA. This led radio frequency chips supply disruption to its two customers, Nokia and Ericsson. After that fire, Nokia promptly worked with its backup suppliers and recovered from this major disruption. However, Ericsson failed to find a new chip supplier. As a result, Ericsson shut down its cell phone business for several months and lost its market share to Nokia. This resulted in a loss of up to $\$ 400$ million in sales and subsequent withdrawal from the mobile phone market [4]. Similar incidents happened to Japanese automobile companies such as Toyota and Honda. In March 2011, after the earthquake and tsunami in Japan, the production and supply of automobile parts in Japan was severely disrupted. This resulted in vast loss to these automobile companies in terms of both profit and reputation [5].

Contrast with unpredictable natural or manmade disasters, large-scale urban activities such as Olympic Games 
and G20 summit are to a large extent predictable. When these activities take place, firms can know when there will be traffic control or government regulations on logistic routes. More predictable risks can also occur due to natural events such as hurricanes. For these risks, historical data are usually available so that we can describe various characteristics including probability, length, and severity level [6-9]. When supply faces more predictable disruption risk, there is a repeated dynamic disruption process from normal supply state to supply disruption and back to normal supply state. This is called dynamic supply disruption where the probability of such risk may be known. To deal with these risks, a firm traditionally can adopt two contingency strategies: holding extra inventory or initiating a backup supplier. Several efficient contingency strategies have been employed in business practice to mitigate the risk from such dynamic supply disruption. For example, Delphi implemented an efficient hybrid contingency strategy in its supply chains during the 2008 Olympic Games in Beijing [3]. Under this strategy, some core technology was transferred to a Virtual Dual Production-Inventory System (VDPIS) and a trade-off was reached between safety inventory and virtual dual sourcing to address this risk of dynamic disruption. In this way, Delphi kept its supply chains lean while minimizing supplier disruption risks.

However, in Delphi's hybrid contingency strategy, there are several operational questions remaining to be answered. What is the best "order-up-level" inventory policy when a firm is informed about the start time and duration of a major event? How much safety inventory should the firm hold? When should the firm transfer its core technology to its VDPIS? How would the transfer delay of its core technology to VDPIS affect its performance?

To answer these questions, this study models a virtual dual-sourcing production-inventory system under predictable and repeated dynamic disruption risks. This system consists of a raw material supplier, a manufacturer, and a virtual dual-sourcing contingency supplier. The manufacturer needs to determine its production, procurement, and inventory plan. When its supplier is interrupted, the manufacturer may need to adjust its plan and work with the contingency supplier. Different from earlier studies, this study considered a scenario of dynamic supply disruption where the start time and duration of a supply disruption and the consequent core technology transfer are interacted with the manufacturer's "order-up-level" inventory polices. Hence, first, our research contributes to the literature on supply chain disruption management by incorporating all these issues simultaneously. Second, we build a system dynamics model to simulate this disrupted supply chain and obtain an approximately optimal order-up-to level inventory policy. Our main research findings are as follows. First, we show that the virtual dual production-inventory strategy can be the optimal contingency policy to deal with supplier dynamic disruption risks. Second, for disruption risk with low frequency and long duration, it is optimal for the manufacturer to increase the safety inventory level before the disruption. Otherwise, it is optimal to increase the safety inventory level in every cycle. Third, with sensitivity analysis, we obtain several managerial implications on how companies mitigate the risks from dynamic supply disruptions due to a relatively predictable event.

The structure of the rest of this paper is described as follows. The related literature is reviewed in Section 2. The model is formulated and described in Section 3. In Section 4, we build and describe the system dynamics simulation model. Next, in Section 5, we present the simulation and sensitivity analysis results. In Section 6, we discuss the results and their managerial insights. Finally, in Section 7, we summarize the paper and present potential future research avenues.

\section{Literature Review}

Our research relates to the research streams of production and distribution planning, disruption management, and dynamic supply disruption, which are reviewed below.

\subsection{Production and Distribution Planning. To deal with} supply or demand uncertainties, Lalmazloumian et al. [10] classified the sources of uncertainty into the three categories of supply, manufacturing, and demand. Successful supply chain management requires an integrated and coordinated planning among different stages such as production and distribution. Several researchers and practitioners have focused on dual-channel supply chains during the last decade. For example, Huang et al. [11] analysed centralized and decentralized production and pricing decisions in a twoechelon dual-channel supply chain model under demand disruption. Panda et al. [12] examined pricing and shipment policies in a dual-channel supply chain for products with decreasing unit cost. Yan et al. [13] developed a two-period game-theoretic model to investigate the effect of product durability on dual-channel operations. Their analysis revealed the effects of product compatibility on the successful operation of dual-channel supply chain and product distribution strategy. The latest research on dual-channel supply chain extends to different areas including product distribution strategy [14], quality improvement [15], price and delivery-time dependent stochastic demand [16], carbon emissions tax, and demand uncertainty [16]. Although there are a few articles in the literature on dual-channel supply chains, few of them have considered the effect of dynamic supply disruption.

2.2. Disruption Management. Supply risk is defined as "the probability of an incident associated with inbound supply from individual supplier failures or the supply market occurring, in which its outcomes result in the inability of the purchasing firm to meet customer demand or cause threats to customer life and safety" [17]. Paul et al. [18] define supply chain risk management (SCRM) as "the management of supply chain risks through coordination or collaboration among the supply chain partners so as to ensure profitability and continuity." Craighead et al. [19] develop a qualitative model relating supply chain design characteristics to mitigation capabilities and propose several propositions to be 
tested quantitatively. Gaonkar and Viswanadham [20] propose a conceptual framework that classify various supply chain risks and various approaches that can be used to deal with those risks. To mitigate the disruption risk, safety inventory and flexible capacity are considered traditional approaches to ensure the smooth operations during supply disruptions. To achieve operational recovery after capacity damage due to major disruption, some studies argue for capacity flexibility to buffer supply chain disruption risk. For example, Bao and Ji [21] propose an analysis framework to disruption management, but they focus mainly on supply chain capacity. Paul and Rahman [22] develop a quantitative and simulation model for managing sudden supply delay with fuzzy demand and safety stock that affect the retailers' economic order quantity model. They find that safety stock plays an important role in recovery from sudden supply delays, and there is a trade-off between backorder and lost sales costs in the recovery plan. When demand is stochastic, optimal inventory policy is the key to manage safety stock $[23,24]$.

However, with decreasing product life cycle and/or increasing product variety, safety inventory may lead to high inventory cost. This is because that the use of a sole supplier can reduce cost but can cause problems under demand variations and disruptions. Billington et al. [25] describe how HP used its manufacturing facilities in Washington State and Singapore to produce printers. HP used the Singapore plant to meet the base demand and use the Washington State plant to meet the above base demand. Such a flexible supply base is beneficial when disruptions occur. Therefore, using backup suppliers can increase supply chain flexibility. To mitigate supply chain disruption risks, Baghalian et al. [26] propose robust supply chain network design where a firm implements strategic inventory or flexible supply base [27]. Paul et al. [28] considered real-time disruption management for a two-stage batch productioninventory system with reliability considerations. In their system, the production may be disrupted, for a given period of time, either at one or both stages, and a recovery plan in real time for a single occurrence of disruption and multiple disruptions at either stage was developed. Later, Paul et al. [29] considered a three-stage supply chain network, with multiple manufacturing plants, distribution centers, and retailers, and developed a quantitative model for disruption mitigation in a supply chain. Furthermore, Paul et al. [30] considered another scenario on sudden transportation disruptions in supply chains under delivery delay and quantity loss, which is very like the condition in our study. They developed a model to generate a recovery plan after a sudden disruption occurrence, helping supply chain managers minimize the negative impacts of the disruption.

To manage risk and disruption in production-inventory and supply chain systems, Paul et al. [18] presented a literature review and developed a disruption recovery model for an imperfect single-stage production-inventory system. The system may unexpectedly face either a single disruption or a mix of multiple dependent and/or independent disruptions, and it formulated a mathematical model for rescheduling the production plan, after the occurrence of a single disruption, which maximizes the total profit during the recovery time window. To manage disruption in an imperfect production-inventory system, Paul et al. [18] developed a disruption recovery model for the system which may unexpectedly face either a single disruption or a mix of multiple dependent and/or independent disruptions and formulated a mathematical model for rescheduling the production plan, after the occurrence of disruption. More recently, Paul and Chowdhury [2] developed a recovery model relating to a revised production plan to deal with the challenge for the manufacturers of high-demand and most essential items during the global pandemic of COVID-19. In this situation, the demand of the essential products increases expressively and the supply of the raw materials decreases considerably with a constraint of production capacity. These dual disruptions impact the production process suddenly, and the process can collapse without immediate and necessary actions. They used a mathematical modeling approach to develop a production recovery model for a high-demand and essential item during the COVID-19 and analysed the properties of the recovery plan.

2.3. Dynamic Supply Disruption. To mitigate supply disruption risk, Sawik [31] considered dual-source procurement strategy. In this virtual dual-sourcing strategy, when a firm's supplier experiences a disruption, the firm can transfer its key design information to a qualified backup supplier during the disruption. Fujimoto and Park [32] argued that when the concentration of suppliers' production technology is relatively high, it will be difficult for manufacturers to find backup suppliers for emergency procurement after a supply disruption. When the transfer of core technology of products is high, a virtual subcontractor can deal with the supply disruption. Thus, virtual dual-source procurement can be effective. Zhang et al. [3] proposed a comprehensive emergency management framework where two contingency strategies of robust inventory control and virtual dual sourcing are integrated. More recently, Zhang et al. [33] studied the contingency polices and operations for supply chain disruptions under changing demand. Although this study integrated safety inventory control and core technology transfer to VDPIS tactically and operationally, it is limited to a scenario of single supply disruption. Therefore, there is a research need to study dynamic supply disruptions. On the contrary, the existing literature on supply disruption risks typically assume a single supplier and optimize inventory as an emergency strategy for supply disruption risk, where the long-term average inventory cost is the main criterion $[34,35]$. Due to the complexity of this hybrid emergency strategy, it is usually difficult to obtain closed-form solutions. To help this challenge, Forrester [36] proposed system dynamics to simulate complex systems. $\mathrm{Hu}$ and $\mathrm{Li}$ [37] applied system dynamics to production planning of products with short life cycle. However, they omit the issue of supply chain disruption. This is another gap we aim to address in this research.

Motivated by the business practice and related academic research discussed so far, this article models a virtual dual 
sourcing inventory system under dynamic supply disruptions considering safety inventories. The decision variables we considered include the "order-up-to-level" inventory, product inventory at the emergency subcontractor, planned production quantity, and product delivery quantity. Our models are complex, but by jointly considering these different issues, they can provide improvements over existing models in the literature.

In short, our study makes several contributions to the literature. First, we developed a hybrid emergency strategy to integrate safety inventory control and virtual dual-source procurement to optimize the safety inventory level and the time delay for the core technology to be transferred to VDPIS. Second, we employed system dynamics to simulate a complex VDPIS under dynamic supply disruptions. This can avoid the problem of unable to obtain closed-form optimal solutions to such a complex system. Third, our results reveal the interactions among the "order-up-level" inventory polices, the duration and start time of supply disruptions, and the core technology transfer to VDPIS. Overall, this study provides managerial insights on how to mitigate the risks from dynamic supply disruptions due to a major event.

\section{Model Description and Formulation}

3.1. Model Description. In this paper, we model a virtual dual-source production inventory system consisting of a raw material supplier, a manufacturer, and an emergency subcontractor that can provide alternative supply in the event of supply disruption. Figure 1 illustrates the system, which is a supply chain network with multiple echelons. When confronted with dynamic supply disruption, the manufacturer can initiate a virtual production through a subcontractor or backup supplier and transfer the product core technology to the subcontractor. When supply disruption is over, the manufacturer will return to normal operations. In short, this system adopts a hybrid emergency strategy combining inventory control with virtual dualsource procurement.

The manufacturer needs to determine the quantity of raw materials, the production plan, and the inventory level. When a dynamic supply disruption occurs, the manufacturer initiates an emergency production and inventory plan. Here, the dynamic supply disruption means the supply of raw materials, components, and finish products is disrupted during a large-scale event at observable period and recovers once the event is over. And the cycle may repeat itself in the future. In other words, a disruption cycle lasts from when the system transforms from the normal supply state to supply disruption state and when it recovers back to the normal supply state.

We assume that the observable duration of the dynamic supply disruption is $n$ periods. We use $x_{i}$ to indicate the supply status. When $x_{i}=0$, the supply of raw materials or products is normal. When $x_{i}=1$, the supply of raw materials or products is disrupted. Similarly, we use $y_{i}$ to indicate the order delivery status. When $y_{i}=0$, it means that an order is normally delivered. When $y_{i}=1$, it means that an order delivery is disrupted.

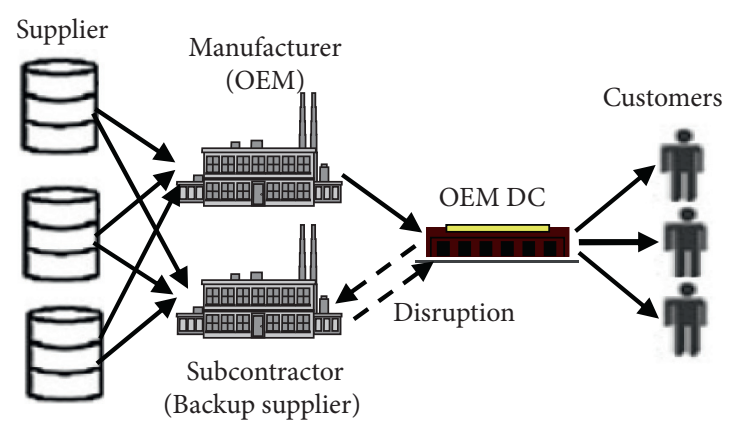

Figure 1: The virtual dual-source production inventory system.

3.2. Model Formulation. All the indices, parameters, and decision variables involved are summarized as follows. The corresponding variables are denoted with superscripts $i=0,1, \ldots, n$.

\subsubsection{Indices}

(i) $i$ denotes the periods, $i=0,1, \ldots, n$.

(ii) $j$ denotes the supply disruption numbers, $j=1,2, \ldots, J$. It denotes the numbers of supply disruptions from the normal supply state to supply disruption state then back to the normal state.

(iii) $k$ denotes the duration of supply disruption, $k=1,2, \ldots, K$.

(iv) $m$ denotes times of supply disruption in the event's horizon, $m=1,2, \ldots, M$.

\subsubsection{Deterministic Parameters}

(i) $h_{r}$ : holding cost of the raw materials

(ii) $C_{m}$ : unit manufacturing cost of the manufacturer

(iii) $h_{m}$ : holding cost of the finished products of the manufacturer

(iv) $C_{S}$ : unit manufacturing cost of the emergency subcontractor

(v) $p$ : unit penalty cost for product shortage

(vi) $r$ : the unit sale price of the product

\subsubsection{Uncertain Parameters}

(i) $T_{m}$ : expected inventory adjustment time. It denotes the time duration it takes for the manufacturer to change from the existing inventory to "order-upto-level" inventory.

(ii) $T_{P}$ : production cycle of the manufacturer.

(iii) $T_{Z}$ : time delay (days) for core product technology to transfer to the emergency subcontractor.

(iv) $T_{S}$ : production cycle (weekly) of the emergency subcontractor.

(v) $d_{i}$ : demand rate (weekly) at period $i, i=0,1, \ldots, n$.

(vi) $d_{i}^{\prime}$ : forecasted demand rate (weekly) at period $i$, $i=0,1, \ldots, n$. 
(vii) $v_{i}$ : lost sales rate (weekly) due to supply disruption at period $i, i=0,1, \ldots, n$.

\subsubsection{Decision Variables}

(i) $\mathrm{IR}_{i}$ : raw materials' inventory of the manufacturer at period $i, i=0,1, \ldots, n$

(ii) $\mathrm{IM}_{i}$ : product inventory at the manufacturer at period $i, i=0,1, \ldots, n$

(iii) $\mathrm{OUL}_{i}$ : “order-up-to-level" inventory at period $i, i=0,1, \ldots, n$

(iv) IS $_{i}$ : product inventory of the emergency subcontractor at period $i, i=0,1, \ldots, n$

(v) $Q_{E}$ : the quantity to order from the emergency subcontractor

(vi) $w_{i}^{m}$ : the quantity of production planned at the manufacturer at period $i, i=0,1, \ldots, n$

(vii) $w_{i}^{s}$ : the quantity of product planned at the emergency subcontractor at period $i, i=0,1, \ldots, n$

(viii) $q_{i}^{m}$ : the delivery quantity of product from the manufacture at period $i, i=0,1, \ldots, n$

(ix) $q_{i}^{s}$ : the delivery quantity of product from the emergency subcontractor at period $i, i=0,1, \ldots, n$

3.2.5. The Objective Function. The total inventory of finished products held by the manufacturer in period $i$ is $I_{i}=\mathrm{IM}_{i}+$ $\mathrm{IS}_{i}$, which is equal to the inventory at the manufacturer plus the inventory at the emergency subcontractor. Thus, $I_{i}=0$ when $y_{i}=0$ indicates that the manufacturer normally delivers according to market orders. When a supply disruption occurs for a long time, the supply status of the raw materials will change from normal to disruption. This leads to the disruption in the manufacturer's product delivery. The cumulative market share loss rate of the manufacturer in time $t$ is

$$
v_{n}=a \cdot \sum_{i=0}^{n} y_{i}+b \cdot\left(\sum_{j=0}^{n} y_{i}\right)^{2}+c
$$

where the term $\sum_{i=0}^{n} y_{i}$ represents the cumulative loss rate of the product delivery disruption at period $t, a$ is the conversion coefficient of this loss rate, the term $\left(\sum_{j=0}^{n} y_{i}\right)^{2}$ represents the cumulative market loss rate caused by frequent disruptions, $b$ is the coefficient between this cumulative market loss rate and the market loss caused by the number of disruptions, and $c$ denotes the error coefficient of market loss caused by the disruption between the two cases. When the manufacturer's product delivery is disrupted, the more disruptions occur in the limited market capacity, the higher the market loss rate is.

It is assumed that the market demand at time $i$ follows the normal distribution with mean $d_{i}$ and standard deviation $\sigma_{i}$. For simplicity, suppose the information's delay period is 3 . It means that the manufacturer's product delivery status undergoes a process from the normal state to disruption state and thereafter from the recovery state to normal state.
Thus, the information's delay is a period of 3 . In this period, the market demand of the product in the lead time of delivery is as follows:

$$
\begin{aligned}
d_{0}^{\prime} & =d_{0}, \\
d_{i}^{\prime} & =\left[d_{i-1}^{\prime}+\frac{\left(d_{i-1}-d_{i-1}^{\prime}\right)}{3}\right], \\
L & =\frac{\left[d_{i-1}^{\prime}+\left(\left(d_{i-1}-d_{i-1}^{\prime}\right) / 3\right)\right]}{d_{i}} .
\end{aligned}
$$

In (2), the forecast rate at initial period $(i=0)$ (weekly) is equal to the demand rate at that period. In (4), $L$ is the lead time of manufacture's delivery, which depends on the forecast rate at period $i$ (weekly) and demand rate at that period. Thus, the manufacturer's "order-up-to-level" inventory is

$$
\mathrm{OUL}_{i}=d_{i}^{\prime}+\mathrm{ss}
$$

where the safety inventory ss for the manufacturer to cope with supply uncertainties is related to the expected turnover service level CSL of their customers. That is, $s=F_{s}^{-1}(\mathrm{CSL}) \times \sigma_{L}$, where $\sigma_{L}=\sqrt{L} \sigma_{i}$. Moreover, we assume that the start-up cost of a virtual production line contract between the manufacturer and the virtual emergency production supplier is $B(\beta)$. This means that the manufacturer is willing to pay for risk prevention in advance for possible disruptions, which is a function of sales $\beta$ during the entire observation period:

$$
B(\beta)=\beta \cdot E\left(r \cdot \sum_{i=0}^{n}\left(q_{i}^{m}+q_{i}^{s}\right)\right),
$$

where $r$ is the unit sale price of the product and $\sum_{i=0}^{n}\left(q_{i}^{m}+\right.$ $\left.q_{i}^{s}\right)$ is the sum of the delivery quantity of product from the manufacture at period $i$ and the delivery quantity of product from the emergency subcontractor at period $i$. Hence, $E(r$. $\left.\sum_{i=0}^{n}\left(q_{i}^{m}+q_{i}^{s}\right)\right)$ means the expectation of the product unit sale price.

3.3. Model Formulation. Under dynamic supply disruptions, there are three scenarios that affect the weekly production and the product delivery amount from the manufacturer to the emergency subcontractor, as described below. Note that, for simplicity, we assume a period to be a week.

(1) In the normal delivery state, $y_{i}=0$, and the manufacturer's weekly production and delivery is

$$
\begin{gathered}
w_{i}^{m}=\mathrm{OUL}_{i}-\mathrm{IM}_{i}, \\
q_{i}^{m}=\operatorname{Min}\left(\mathrm{IM}_{i}, d_{i}\right) .
\end{gathered}
$$

(2) In the state of delivery disruption, $y_{i}=1$, and the manufacturer turns to the emergency subcontractor. In practice, when a supply disruption does not affect production yet, the management may be unaware of 
it. Therefore, the emergency strategy of the subcontractor may lag. After several cycle lags, the managers may decide whether to initiate emergency production. Therefore, the model assumes that, in the case of simultaneous delivery and supply disruptions, the production at the emergency subcontractor will be initiated. The weekly production volume and delivery volume from the emergency subcontractor are

$$
\begin{aligned}
w_{i}^{s} & =\operatorname{Min}\left[Q_{E}, \mathrm{OUL}_{i}-\mathrm{IS}_{i}\right] \\
q_{i}^{s} & =\operatorname{Min}\left(\mathrm{IS}_{i}, d_{i}\right) .
\end{aligned}
$$

(3) Suppose at any time $t+1$, when $x_{i}^{t}=1$ and $x_{i}^{t+1}=0$, the manufacturer restores from disruption to resume normal product. Hence, the production at the emergency subcontractor will be stopped. The manufacturer's inventory will also resume normal delivery. At this time, the inventory at the emergency subcontractor is only used to supplement the current part of the bill of arrears until it is exhausted. We call it the recovery process after the disruption. In the recovery process, the production volume and delivery volume from the manufacturer and the emergency subcontractor are

$$
\begin{aligned}
w_{i}^{m} & =\mathrm{OUL}_{i}-\mathrm{IM}_{i}-w_{i}^{s}, \\
w_{i}^{s} & =\operatorname{Min}\left[Q_{E}, \mathrm{OUL}_{i}-\mathrm{IS}_{i}\right], \\
q_{i}^{m} & =\operatorname{Min}\left(\mathrm{IM}_{i}, d_{i}^{\prime}\right), \\
q_{i}^{s} & =\operatorname{Min}\left[\left(d_{i}^{\prime}-q_{i}^{m}\right), \mathrm{IS}_{i}\right] .
\end{aligned}
$$

Considering the three scenarios, we can build the following optimization model for the virtual dual productioninventory system to maximize the total long-term profit and minimize the profit-loss risk:

$$
\begin{aligned}
\pi\left(q_{i}^{m}, q_{i}^{s}, \beta\right) & =E\left\{\sum_{i=0}^{n}\left\{r \cdot\left(q_{i}^{m}+q_{i}^{s}\right)-\left[\left(h_{r} \cdot I R_{i}+c_{m} \cdot w_{i}^{m}+c_{s} \cdot w_{i}^{s}+h_{m} \cdot\left(I M_{i-1}-q_{i}^{m}\right)^{+} h_{s} \cdot\left(I S_{i-1}-q_{i}^{s}\right)^{+}+p\left(d_{i}-q_{i}^{m}-q_{i}^{s}\right)^{+}\right]\right\}+B(\beta)\right\}\right. \\
\Delta_{\pi} & =\sum_{i=0}^{n} \pi\left(q_{i}^{m}, q_{i}^{s}, \beta\right)-\sum_{i=0}^{n} \pi\left(q_{i}^{m}, 0, \beta\right)
\end{aligned}
$$

By maximizing the manufacturer's profit (10) and minimizing long-term profit-loss risk (11), the optimal "order-to-level" inventory $\mathbf{O U L}_{i}^{*}$ of the system and other decision variables such as the quantity to order from the emergency subcontractor $\mathbf{Q}_{\mathrm{E}}$, product inventory at the emergency subcontractor $\mathbf{I S}_{\mathbf{i}}$, the quantity of production planned at the manufacturer $\mathbf{w}_{\mathbf{i}}^{\mathbf{m}}$, the quantity of product planned at the emergency subcontractor $\mathbf{w}_{\mathbf{i}}^{\mathbf{s}}$, the delivery quantity of product from the manufacture at period $\mathbf{q}_{\mathbf{i}}^{\mathbf{m}}$, and the delivery quantity of product from the emergency subcontractor $\mathbf{q}_{\mathbf{i}}^{\mathbf{s}}$ can be obtained.

\section{Simulation}

Due to the complexity of our dynamic optimization model, it is difficult to obtain a closed-form solution. Therefore, we adopt Forrester's system dynamic theory to simulate our VDPIS in these scenarios. We employ VENSIM PLE software package to simulate our system to obtain approximately optimal solutions in these scenarios. The system dynamics model is illustrated in Figure 2. In our simulation model, there are several suppliers $(\mathbf{l}=1,2, \ldots, \mathbf{L})$ to supply raw materials to the manufacturer (or OEM) or the subcontractor (or back-up supplier) to produce their products and then distribute them to different customers $\mathbf{d}_{\mathbf{i}}$ in different periods. This market demand $\mathbf{d}_{\mathbf{i}}$ is an aggregate of all demand in one period. It follows the normal distribution with mean $\mathbf{d}_{\mathbf{i}}$ and standard deviation $\sigma_{\mathrm{i}}$. When the manufacturer's supply is disrupted, the manufacturer will transfer its core technology to the subcontractor and implement the socalled virtual dual-source procurement from the subcontractor and deliver the products to customers via OEM DC. In OEM DC, the safety inventory depends on market demands, product capabilities of the manufacturer and the subcontractor, and the mode of disruption (disruptive times $\mathbf{m}$ and disruptive duration k). Before starting the dynamic system simulation, inputs should be collected from suppliers, subcontractor, DC, and customers. We consider order delivery status, inventory, and the manufacturer's long-term profit of as state variables. We treat the manufacturer inventory sequence and the emergency supplier inventory sequence as the mainstream structures. We treat the supplier's supply flow, market demand loss, and longterm profit as the co-flow structure. Our objective is to maximize the total long-term profit and minimize the profit-loss risk. In the end, we will obtain the outputs including the production planning, OUL inventory, profit, and risk.

Next, we will describe the simulations in detail. In the dynamic system simulation, we use the following function to input the disruption state as a pulse function into the dynamic model to reflect the dynamic disruption of suppliers: PULSETRAIN (initial time, $k, 50, n) . \mathrm{IM}_{i}$ in the theoretical model is manufacturer inventory in the dynamic model. "Manufacturer's weekly production volume" is equivalent to "manufacturer's production forecast" in the system dynamics 


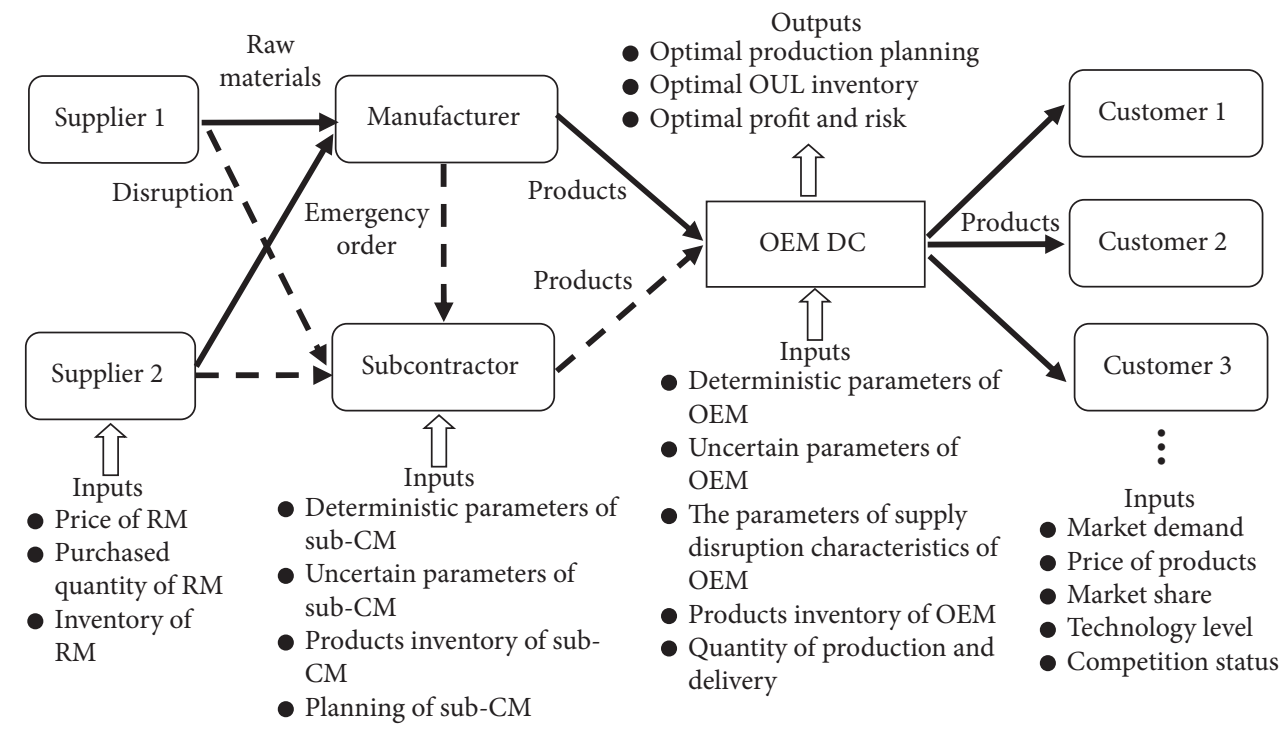

FIgURE 2: The system dynamics simulation model on dynamic supply disruption.

model. And "Safety Inventory Level" is equivalent to "Orderup-to-Level" (OUL).

Based on observations and experiments from the production of car dashboards in an automobile assembly factory, we collected data related to supply disruptions due to the 2008 Olympic Games in Beijing. The data include order delivery status, manufacturer inventory, emergency production supplier inventory, and the manufacturer's long-term profit, which are state variables. Based on the historical demand of the dashboard product, it is assumed that the weekly demand follows the normal distribution $d \sim N\left(80,20^{2}\right)$, where the standard deviation is $\sigma=20$ units and the sales forecast $d_{0}^{\prime}=$ INTEGER (SMOOTH (week demand, 3)). We set $a=$ 0.01 and $b=0.0015$ in (1). Other parameters are set to $h_{r}=0 \$ /$ unit $, \quad n=252, \quad C_{m}=4 \$$ /unit,$\quad h_{m}=0.2 \$$ /unit, $h_{s}=0.8 \$$ /unit, $C_{s}=6 \$$ /unit, $p=0.8 \$$ /unit, $Q_{E}=240$ units, $\beta=0.01, r=20 \$ /$ unit, $T_{m}=3$ units, $T_{z}=3$ units, $T_{P}=1$ unit, and $v_{0}=0$ unit. In addition, in the absence of disruption, we set the manufacturer's safety inventory as $\mathrm{ss}_{0}^{*}=170$ units and the emergency supplier's initial inventory as $\mathrm{IS}_{0}=0$ unit. Here, suppose that the manufacturer employs a strategy of JIT or VMI to its suppliers. Thus, we set $h_{r}=0 \$ /$ unit. When starting a simulation, we set the initial time $=10$ (weeks), the termination time $=52$ (weeks), and the duration step $=1$ (week). After providing all parameter values into our dynamic simulation model, we can obtain results on safety inventory, long-term profit, and profit-loss risk under each of the three scenarios of disruption mode. We also find the impacts of delaying core technology transfer and multicycle inventory control policies on safety inventory and the long-term profit.

\section{Results and Sensitivity Analysis}

5.1. Safety Inventory Policies. The optimal safety inventory ss* can be obtained by the optimal "order-up-to-level" inventory $\mathrm{OUL}_{i}^{*}$, as shown in (5). To analyse the optimal safety inventory polices, we set the three scenarios of dynamic disruption modes as follows:
(1) Disruption mode A $((m, k)=(20,3))$ : it means that the system is disrupted 20 times in one cycle and lasts 3 cycles due to one major event

(2) Disruption mode B $((m, k)=(3,20))$ : it means that the system is disrupted 3 times in one cycle and lasts 20 cycles due to one major event

(3) Disruption mode $C((m, k)=(7,10))$ : it means that the system is disrupted 7 times in one cycle and lasts 10 cycles due to one major event

We set three models according to the times of supply disruption $m$ and the duration of supply disruption $k$ from 3 to 20. Among the three models, mode A has longest times of supply disruption but shortest duration of supply disruption, mode B has its opposite, and mode $C$ has a medium time of supply disruption durations between $\mathrm{A}$ and $\mathrm{B}$. These settings can help figure out which is more impactful on the safety inventory between the times of supply disruption and the duration of supply disruption.

To mitigate the risks from these disruption modes, we suppose that there are three safety inventory policies:

(1) To hold safety inventory level ss* as a normal supply case

(2) To increase the safety inventory level to $2.0 \mathrm{ss} *$ in each cycle with the limit of the manufacturer's production capacity

(3) To increase the safety inventory level to $2.5 \mathrm{ss} *$ before the start of supply disruption

The three scenarios above are based on our observations from industry practices [3, 33]. Running our dynamic system simulations, we obtain the manufacturer's long-term profit under different disruption modes and safety inventory polices, as shown in Figure 3.

As can be seen from Figure 3, the manufacturer's longterm profit under the disruption mode $A$ is highest at the $3^{\text {rd }}$ 
safety inventory policy (vs. the $1^{\text {st }}$ and $2^{\text {nd }}$ ). Thus, the manufacturer should choose to hold safety inventory close to $2.5 \mathrm{ss}^{*}$ under dynamic disruptions with high frequency but short duration. This may occur for large-scale urban activities. Furthermore, the manufacturer should hold its safety inventory before the start of supply disruption. On the contrary, when dynamic disruptions occur at low frequency but long duration (disruption mode B), the manufacturer's long-term profit is highest under the second safety inventory policy. It means that the manufacturer should hold safety inventory closer to $2.0 \mathrm{ss}^{*}$ under dynamic disruptions with low frequency but long duration. This may occur due to natural disasters. Furthermore, it is better for the manufacturer to adopt the multicycle inventory control polices than holding emergency safety inventory in advance. Under the disruption mode $\mathrm{C}$ with medium frequency but duration, the manufacturer's long-term profit is the lowest among the three. This rarely happens. However, if it does, the manufacturer should setup its inventory according a special multicycle inventory control policy. Moreover, in reality, major events such as large-scale urban activities are associated with disruptions of high frequency and short duration. The government usually informs firms in advance about when to open and close public infrastructure such as roads and ports. This helps manufacturers anticipate the disruption mode in advance and plan accordingly.

5.2. Multicycle Inventory Control Policies. For the case of multicycle disruption, for simplicity, we consider two scenarios.

Scenario 1 (Mode A): $(m, k)=(20,3)$. It means that the system is disrupted 20 periods in one cycle and lasts 3 cycles due to one major event.

Scenario 2 (Mode B): $(m, k)=(3,20)$. It means that the system is disrupted 3 periods in one cycle and lasts 20 cycles due to one major event.

In our system dynamics simulation under these two scenarios, we optimize the virtual dual-source purchasing inventory under the multicycle inventory control strategy. We are able to obtain the system's operational performance such as Initiate Emergency Inventory, Consumed Time of Emergency Supplier Inventory, Inventory Cost/Total Cost of Emergency Supplier, Average Order Delivered Rate, Market Loss Rate, and Manufacturer's Long-term Profits, which are summarized in Table 1.

It can be seen from Table 1 that, in the disruption mode of short disruption time and high frequency, it is necessary to transfer the production core technology to the emergency production supplier. The inventory emergency strategy should be waiting for recovery. When the disruption time is long and the frequency is low, the long-term profit of the manufacturer can be increased by up to $25 \%$ by initiating the emergency supplier (vs. the strategy of waiting for recovery). In the case of $[3,20]$, the use of the emergency supplier is 48

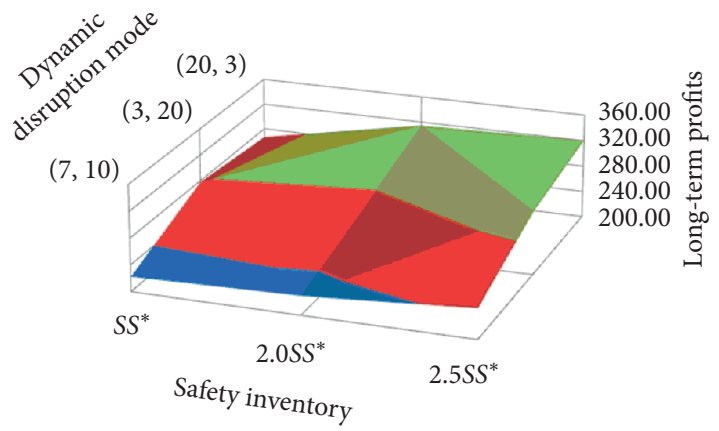

FIgURe 3: The impact of dynamic disruption modes and safety inventory policies on the long-term profit.

weeks, which is shorter than the disruption duration of the whole cycle (60 weeks). This indicates that the implementation of emergency strategy is delayed for a certain time.

Furthermore, we can draw the following management insights. If there are frequent disruptions caused by regulatory measures such as short-term temporary power cuts, the manufacturer can adopt the emergency inventory strategy of waiting for recovery. If the supply is interrupted for a long time due to large-scale activities or events, the manufacturer should start the emergency production inventory strategy.

5.3. Transfer Delay of Core Technologies. Assuming that the delay of the production core technology transfer is $T_{Z}=$ $\{1,3,5,8\}$ and the dynamic disruption is $(m, k)=(6,15)$. Employing dynamic simulations, we find that the core technology transfer delay does impact the emergency supplier inventory (product delivery) and firm profit. Figure 4 illustrates how the delivered quantity from the emergency supplier $q_{i}^{s}$ varies when the dynamic disruption occurs.

Figure 4 shows that the longer the core technology transfer delay, the more quantity to be delivered from the emergency supplier's factory. This is because that the delay will prolong the inventory use cycle of the emergency supplier. This would increase the inventory holding cost and decrease the long-term profit of the manufacturer, as shown in Figure 5.

Based on these results above, we can conclude that the manufacturer can manage their emergency inventory and transfer their product core technology delay according to the supply disruptive duration $k$ and the times $m$. For dynamic disruptions with higher frequency, managers should transfer the production core technology to the emergency supplier to decrease the delivered quantity (or inventory).

5.4. Risks of Long-Term Profit Loss. To estimate the risks of the manufacturer's long-term profit under dynamic supply disruptions, we conducted a sensitivity analysis in terms of the durations and the start times of supply disruptions. Under the dynamic supply disruption portfolio $(m, k)$, we obtain three-dimensional surfaces, as illustrated in Figure 6. 
TABLE 1: Operational performance of the virtual dual-source purchasing inventory system.

\begin{tabular}{lccccccc}
\hline Mode & $m$ & IES? & CT-EI & IC/TC & ADR (\%) & MRL (\%) & MLP \\
\hline$(0,0)$ & 0 & $\mathrm{X}$ & 0 & 0 & 91.0 & 0.30 & 291,003 \\
$(20,1)$ & 60 & $\mathrm{X}$ & 0 & $0 \%$ & 85.5 & 3.20 & 271,612 \\
$(3,20)$ & 60 & 3 & 48 & $35 \%$ & 89.5 & 1.80 & 251,529 \\
$(3,20)$ & 60 & $\mathrm{X}$ & 0 & $0 \%$ & 73.4 & 9.10 & 201,177 \\
\hline
\end{tabular}

Note: $E S=$ emergency supplier; EI = initiate emergency inventory; CT$\mathrm{EI}=$ consumed time of emergency supplier inventory; $\mathrm{IC} / \mathrm{TC}=$ inventory cost/total cost of emergency supplier; $\mathrm{ADR}=$ average order delivered rate; $\mathrm{MLR}=$ market loss rate; MLP = manufacturer's long-term profits.

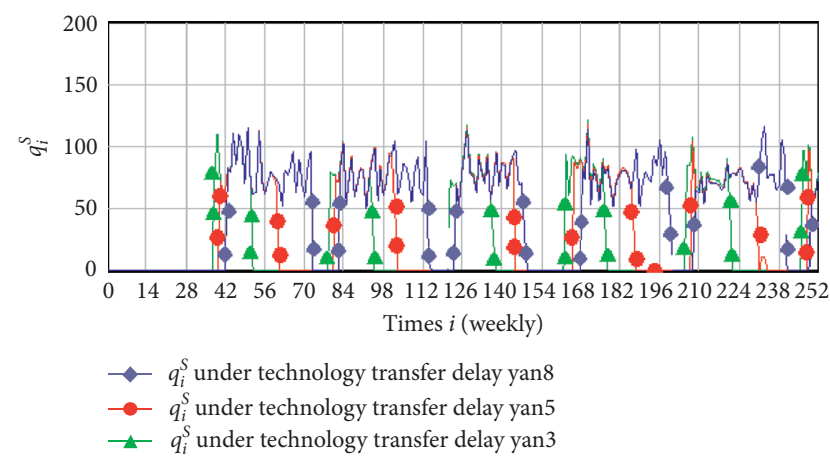

FIGURE 4: The impact of core production technology transfer delay on the emergency inventory.

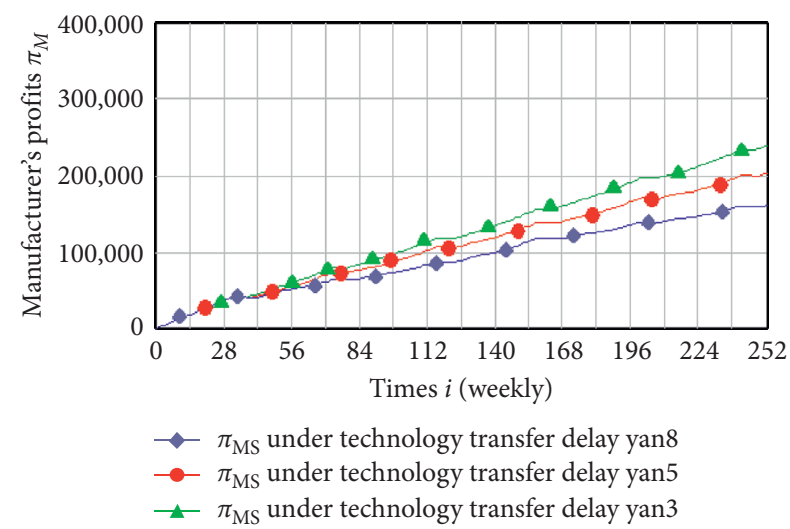

Figure 5: The impact of transfer core technology delay on the manufacturer's long-term profit.

In Figure 6, we can see that when $m$ remains fixed and $k$ increases, the manufacturer's long-term profit loss risk will increase. This implies that the manufacturer will be willing to adopt virtual production contracts to reduce the risk. Therefore, the manufacturer should transfer the production core technology to the emergency supplier earlier when a supply disruption lasts longer.

Moreover, when $k$ is fixed at a small value, the reduction of the manufacturer's long-term profit loss risk changes insignificantly with the increase of dynamic supply disruptive times $m$. This means that the manufacturer is unwilling to pay more for virtual production. Therefore, the long-term profit loss is more sensitive to $k$ than $m$. This finding suggests that when anticipating a long-duration

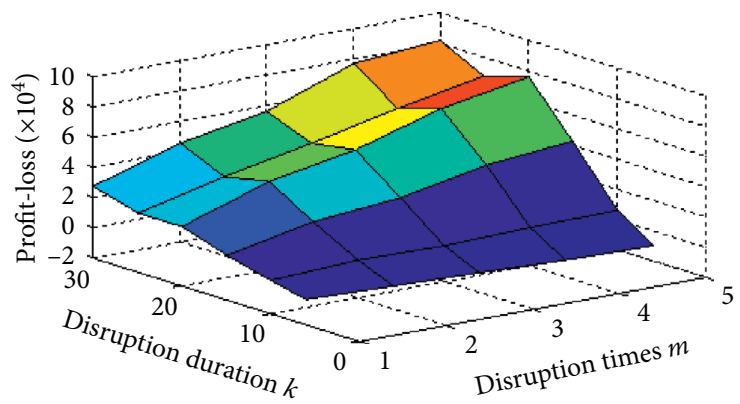

Figure 6: Sensitivity of the manufacturer's profit loss risks to the duration and times of supply disruptions.

supply disruption, the manufacturer transfers the core technology to the emergency supplier. In short, an emergency strategy of virtual dual sourcing is the key to succeed under dynamic supply disruption.

\section{Discussion and Managerial Insights}

In Section 5, we first study safety inventory under the three disruption modes (A, B, and $\mathrm{C}$ ). Our result shows that, under dynamic disruptions with high frequency but short duration (disruption mode $\mathrm{A}$ ), the manufacturer should hold its safety inventory at a level closer to $2.5 \mathrm{ss}^{*}$ before the start of supply disruption to maximize the long-term profit and minimize the profit-loss risk. Under dynamic disruptions occurring at low frequency but long duration (disruption mode B), the manufacturer should hold safety inventory at level of closer to 2.0ss*. Finally, under disruption mode $\mathrm{C}$ with medium frequency and duration, the manufacturer's long-term profit is the lowest among the three. In practice, disruption mode $B$ occurs due to natural disasters, disruption mode $\mathrm{C}$ rarely happens, and disruption mode A may occur for large-scale urban activities. Furthermore, under disruption mode A, our results indicate that the government should inform firms in advance about when to open and close public infrastructure such as roads and ports. This helps manufacturers plan accordingly.

Second, our results show that, under the disruption mode of short disruption time and high frequency (disruption mode $\mathrm{A}$ ), the manufacturer's optimal policy is to initiate the emergency supplier and the strategy of waiting for recovery. In this condition, it is necessary for the manufacturer to transfer the production core technology to the emergency supplier. Under the disruption mode of long disruption time and low frequency (disruption mode B), the delivery time can be decreased by the use of the emergency supplier. Therefore, we can draw the following management insights. If there are frequent disruptions caused by regulatory measures such as short-term temporary power cuts, the manufacturer can adopt the emergency inventory strategy waiting for recovery. If the supply is interrupted for a long time due to large-scale activities or events, the manufacturer should start the emergency production inventory strategy. This result can help the manufacturer decide on when it should choose its emergency inventory 
strategy waiting for recovery or the emergency production inventory strategy.

Third, we analyse the impact of core technology transfer delay on the manufacturer's delivery after the manufacturer adopts the strategy of the emergency production inventory strategy. Our result shows that the longer the core technology transfer delay is, the more the quantity to be delivered from the emergency supplier's factory. Therefore, for dynamic disruptions with higher frequency, managers should transfer the production core technology to the emergency supplier to decrease inventory.

Last, we analyse the impact of the disruption frequency and duration on the manufacturer's risks of long-term profit loss. Our results show that when disruption frequency $m$ remains fixed but disruption duration $k$ increases, the manufacturer's long-term profit loss risk will increase. Hence, the manufacturer should adopt a virtual production contact and transfer its core production technology to the emergency supplier earlier when a supply disruption lasts longer to reduce the risk of long-term profit loss. Moreover, when disruption duration $k$ is fixed at a small value, the reduction of the manufacturer's long-term profit loss risk changes insignificantly with the dynamic supply disruptive duration $m$. This implies when anticipating a long-duration supply disruption, the manufacturer should transfer the core technology to the emergency supplier as early as possible and an emergency strategy of virtual dual sourcing is an optimal policy to reduce the risk of long-term profit loss.

\section{Conclusions and Future Research}

When major urban events such as Olympic Games and G20 Summit are held in a city, they result in dynamic supply disruptions and challenge firms' supply chain management. In this paper, we investigate how to deal with the dynamic disruption risks in a production inventory system consisting of a raw material supplier, a manufacturer, and a virtual dual-source emergency supplier. We first study a hybrid emergency strategy of robust "order-up-level" inventory policy and virtual dual-sourcing production. Considering three scenarios, we build a dynamic optimization model for this virtual dual production-inventory system to maximize the total long-term profit and minimize the profit loss risk. We then use system dynamics simulation to obtain approximately optimal solutions.

By using the system dynamics simulation software of VENSIM PL, we investigate the impacts of different dynamic disruption models on the "order-up-level" inventory and the impact of core production technology transfer delay on the emergency safety inventory. We also conduct sensitivity analysis to check how the manufacturer's profit loss risk is impacted by the durations and start times of supply disruptions. Our results show that the manufacturer's longterm profit under disruptions of high frequency but short duration (e.g., disruptions due to large-scale urban activities) is the highest. Hence, for such disruptions, it is optimal for the manufacturer to increase the safety inventory level in each disruption cycle. On the contrary, when dynamic disruption takes place at low frequency but long duration (e.g., disruption due to a natural disaster), the manufacturer's long-term profit is the highest under the second safety inventory policy. In other words, for such disruptions, it is optimal for the manufacturer to adopt the emergency strategy of raising the safety inventory level before disruption.

Moreover, our results show that the longer the core production technology transfer delay is, the more the quantity to be delivered from the emergency supplier. The delay of technology transfer also has a greater impact on the profit in the recovery cycle after disruption. The disruption duration affects the profit loss risk more than the number of disruptions. Under dynamic disruptions with higher frequency, managers should transfer their core technology to reduce the delivered quantity (or inventory). In addition, the virtual dual-source production inventory strategy is the optimal under dynamic disruption risk. Therefore, an emergency strategy of virtual dual sourcing is the key to manage dynamic supply disruptions.

In this study, based on industry observations, we limit ourselves to three safety inventory polices according to the three possible scenarios of supply disruptions. Future research can identify and investigate other possible safety inventory policies. It is also worthwhile to make our research more targeted to the COVID-19 global pandemic. Besides, flexible capacity of the manufacturer and emergency supplier is a promising strategy to deal with dynamic supply disruption. Hence, future research can be carried out on the trade-off between flexible capacity and cost under dynamic supply disruption risks. Last but not least, in this paper, we have conducted several numerical examples and their sensitivity analysis. However, to make these results more realistic, in future research, more numerical examples and sensitivity analysis can be carried out relaxing one or more assumptions in this paper.

\section{Data Availability}

The data used to support the findings of the study are available from the corresponding author upon request.

\section{Conflicts of Interest}

The authors declare that they have no conflicts of interest.

\section{Acknowledgments}

This research was supported by the National Social Science Foundation of China (no. 15CGL008), Plateau Discipline of Business Administration Construction Fund of Shanghai Lixin University of Accounting and Finance, and Visiting Academic Fellow Program in Shanghai Lixin University of Accounting (LX-HR-20190312). Dr. Zhu was supported by the National Natural Science Foundation of China (no. 71771188) and Fundamental Research Funds for the Central Universities (nos. JBK18JYT02, JBK1805008, and JBK190504). 


\section{References}

[1] K. Govindan, H. Mina, and B. Alavi, "A decision support system for demand management in healthcare supply chains considering the epidemic outbreaks: a case study of coronavirus disease 2019 (COVID-19)," Transportation Research Part E: Logistics and Transportation Review, vol. 138, p. 101967, 2020.

[2] S. K. Paul and P. Chowdhury, "A production recovery plan in manufacturing supply chains for a high-demand item during COVID-19," International Journal of Physical Distribution \& Logistics Management, 2020, In press.

[3] Y. Zhang, J. Long, and C. Shi, "A comprehensive contingency management framework for supply chain disruption risk management," International Journal of Automation and Logistics, vol. 1, no. 4, pp. 343-369, 2015.

[4] H. L. Lee, "The triple-A supply chain," Harvard Business Review, vol. 82, no. 10, pp. 102-157, 2004.

[5] M. Ramsey and S. Moffett, "Japan parts shortage hits auto makers," The Wall Street Journal, vol. B1, 2011.

[6] S. Li, Y. He, and L. Chen, "Dynamic strategies for supply disruptions in production-inventory systems," International Journal of Production Economics, vol. 194, pp. 88-101, 2017.

[7] C. Martinez, "Supply chain risks, resilience and firm performance: an empirical study," $\mathrm{Ph}$. D thesis, University of Maryland, College Park, MD, USA, 2018.

[8] Y. He, S. Li, H. Xu, and C. Shi, "An in-depth analysis of contingent sourcing strategy for handling supply disruptions," IEEE Transactions on Engineering Management, vol. 67, no. 1, pp. 201-219, 2020.

[9] S. Ma, Y. He, and R. Gu, "Dynamic generic and brand advertising decisions under supply disruption," International Journal of Production Research, pp. 1-25, 2020.

[10] M. Lalmazloumian, K. Y. Wong, K. Govindan, and D. Kannan, "A robust optimization model for agile and buildto-order supply chain planning under uncertainties," Annals of Operations Research, vol. 240, no. 2, pp. 435-470, 2016.

[11] S. Huang, C. Yang, and X. Zhang, "Pricing and production decisions in dual-channel supply chains with demand disruptions," Computers \& Industrial Engineering, vol. 62, no. 1, pp. 70-83, 2012.

[12] S. Panda, N. M. Modak, S. S. Sana, and M. Basu, "Pricing and replenishment policies in dual-channel supply chain under continuous unit cost decrease," Applied Mathematics and Computation, vol. 256, pp. 913-929, 2015.

[13] W. Yan, Y. Xiong, J. Chu, G. Li, and Z. Xiong, "Clicks versus Bricks: the role of durability in marketing channel strategy of durable goods manufacturers," European Journal of Operational Research, vol. 265, no. 3, pp. 909-918, 2018.

[14] K. Matsui, "Asymmetric product distribution between symmetric manufacturers using dual-channel supply chains," European Journal of Operational Research, vol. 248, no. 2, pp. 646-657, 2016.

[15] X. Chen, H. Zhang, M. Zhang, and J. Chen, "Optimal decisions in a retailer Stackelberg supply chain," International Journal of Production Economics, vol. 187, pp. 260-270, 2017.

[16] N. M. Modak and P. Kelle, "Managing a dual-channel supply chain under price and delivery-time dependent stochastic demand," European Journal of Operational Research, vol. 272, no. 1, pp. 147-161, 2019.

[17] G. A. Zsidisin, "A grounded definition of supply risk," Journal of Purchasing and Supply Management, vol. 9, no. 5-6, pp. 217-224, 2003.
[18] S. K. Paul, R. Sarker, and D. Essam, "Managing disruption in an imperfect production-inventory system," Computers \& Industrial Engineering, vol. 84, pp. 101-112, 2015.

[19] C. W. Craighead, J. Blackhurst, M. J. Rungtusanatham, and R. B. Handfield, "The severity of supply chain disruptions: design characteristics and mitigation capabilities," Decision Sciences, vol. 38, no. 1, pp. 131-156, 2007.

[20] R. S. Gaonkar and N. Viswanadham, "Analytical framework for the management of risk in supply chains," IEEE Transactions on Automation Science and Engineering, vol. 4, no. 2, pp. 265-273, 2007.

[21] X. Bao and J. Ji, "Disrupting capacity management of service operation system under decision-maker's risk attitude," Journal of Systems \& Management, vol. 18, no. 5, pp. 555-561, 2009.

[22] S. K. Paul and S. Rahman, "A quantitative and simulation model for managing sudden supply delay with fuzzy demand and safety stock," International Journal of Production Research, vol. 56, no. 13, pp. 4377-4395, 2018.

[23] N. M. Modak, S. Panda, and S. S. Sana, "Optimal inventory policy in hospitals: a supply chain model," Revista de La Real Academia de Ciencias Exactas Fisicas Y Naturales Serie A-Matematicas, vol. 114, no. 3, 2020.

[24] S. Sinha, N. M. Modak, and S. S. Sana, "An entropic order quantity inventory model for quality assessment considering price sensitive demand," Opsearch, vol. 57, no. 1, pp. 88-103, 2020.

[25] C. Billington, B. Johnson, and A. Triantis, "A real options perspective on supply chain management in high Technology1," Journal of Applied Corporate Finance, vol. 15, no. 2, pp. 32-43, 2002.

[26] A. Baghalian, S. Rezapour, and R. Z. Farahani, "Robust supply chain network design with service level against disruptions and demand uncertainties: a real-life case," European Journal of Operational Research, vol. 227, no. 1, pp. 199-215, 2013.

[27] C. S. Tang, "Perspectives in supply chain risk management," International Journal of Production Economics, vol. 103, no. 2, pp. 451-488, 2006.

[28] S. K. Paul, R. Sarker, and D. Essam, "Real time disruption management for a two-stage batch production-inventory system with reliability considerations," European Journal of Operational Research, vol. 237, no. 1, pp. 113-128, 2014.

[29] S. K. Paul, R. Sarker, and D. Essam, "A quantitative model for disruption mitigation in a supply chain," European Journal of Operational Research, vol. 257, no. 3, pp. 881-895, 2017.

[30] S. K. Paul, S. Asian, M. Goh, and S. A. Torabi, "Managing sudden transportation disruptions in supply chains under delivery delay and quantity loss," Annals of Operations Research, vol. 273, no. 1-2, pp. 783-814, 2019.

[31] T. Sawik, "Joint supplier selection and scheduling of customer orders under disruption risks: single vs. dual sourcing," Omega, vol. 43, pp. 83-95, 2014.

[32] T. Fujimoto and Y. W. Park, "Balancing supply chain competitiveness and robustness through "virtual dual sourcing": lessons from the Great East Japan Earthquake," International Journal of Production Economics, vol. 147, pp. 429-436, 2014.

[33] J. Zhang, Y. Liu, Y. Zhao, and T. Deng, "Emergency evacuation problem for a multi-source and multi-destination transportation network: mathematical model and case study," Annals of Operations Research, vol. 29, no. (1-2), pp. 11531181, 2018.

[34] B. Atasoy, R. Güllü, and T. Tan, "Optimal inventory policies with non-stationary supply disruptions and advance supply 
information," Decision Support Systems, vol. 53, no. 2, pp. 269-281, 2012.

[35] A. M. Ross, Y. Rong, and L. V. Snyder, "Supply disruptions with time-dependent parameters," Computers \& Operations Research, vol. 35, no. 11, pp. 3504-3529, 2008.

[36] J. W. Forrester, "Industrial dynamics. a major breakthrough for decision makers," Harvard Business Review, vol. 36, no. 4, pp. 37-66, 1958.

[37] H. Hu and Y. Li, "Dynamic batch production planning for short Life cycle products considering remanufacturing and replaceable product demand," System Engineering Theory and Practice, vol. 12, pp. 76-84, 2007. 